\title{
ELIMED: MEDICAL APPLICATION AT ELI-BEAMLINES. STATUS OF THE COLLABORATION AND FIRST RESULTS
} \author{
Mariapompea CutroneO $^{a, f}$, Antonella Tramontana ${ }^{a}$ \\ a INFN-LNS, Catania, Italy \\ ${ }^{b}$ Institute of Physics of the ASCR, ELI-Beamlines Project, Prague, Czech Republic \\ c INFN-LNL, Legnaro, Italy \\ ${ }^{d}$ University of Catania, Catania, Italy \\ e Centro Studi e Ricerche E. Fermi, Roma, Italy \\ $f$ Dip.to di Fisica, University of Messina, Messina, Italy \\ * corresponding author: schillacif@lns.infn.it
}

Francesco Schillaci ${ }^{a, *}$, Giuseppe A. P. Cirrone ${ }^{a, b}$, George Korn $^{b}$, Mario Maggiore $^{c, b}$, Daniele Margarone ${ }^{b}$, Luciano Calabretta ${ }^{a}$, Salvatore Cavallaro $^{a}$, Giacomo Cuttone ${ }^{a}$, Santo Gammino ${ }^{a}$, Josef Krasa ${ }^{b}$, Jan ProkUpeK ${ }^{b}$, Andriy Velyhan $^{b}$, Marcella Renis ${ }^{d}$, Francesco Romano $^{a, e}$, Barbara Tomasello ${ }^{d}$, Lorenzo Torrisi ${ }^{a, f}$,

\begin{abstract}
ELI-Beamlines is one of the four pillars of the ELI (Extreme Light Infrastructure) pan-European project. It will be an ultrahigh-intensity, high repetition-rate, femtosecond laser facility whose main goal is to generate and apply high-brightness X-ray sources and accelerated charged particles. In particular, medical applications are treated by the ELIMED task force, which has been launched by collaboration between ELI and INFN researchers. ELIMED aims to demonstrate the clinical applicability of laser accelerated ions. In this article, the state of the ELIMED project and the first scientific results are reported. The design and realisation of a preliminary beam handling system and of an advanced spectrometer for diagnostics of high energy (multi-MeV) laser-accelerated ion beams will also be briefly presented.
\end{abstract}

KEYworDs: laser acceleration; cancer treatment; Thomson Parabola; beam handling.

\section{INTRODUCTION}

Collimated multi-MeV proton beams can be generated by irradiating thin solid foils with ultraintense $\left(10^{18} \mathrm{~W} / \mathrm{cm}^{2}\right)$ short-pulse (30 fsec-10 psec) lasers. Nowadays, the highest proton energies with best characteristics have been reached using the so called Target Normal Sheath Acceleration (TNSA) mechanism [1]. In this regime, high-energy ( $\mathrm{MeV}$ range) electrons are generated at the target front side by hot electron formation. The electrons have a mean free-path larger than the target thickness [2 4, which is in the range of a few hundred micron or smaller. As a consequence, the electrons can cross the target producing an intense electrostatic field (in the range of $\mathrm{TV} / \mathrm{m}$ ) due to the charge imbalance between positive ions at rest on the target and the electron sheath expanding at its rear surface, in the laser incidence direction [5]. Laserbased accelerators can have performances comparable with, or maybe higher than, huge and expensive conventional accelerators, and the device size could end up more compact $(1 / 10-1 / 100)$ than present facilities 6 . It must be stressed that laser-produced proton beams show a large divergence angle [7] and energy-spread (up to $100 \%$ ) with a cut-off at the maximum energy. The characteristics depend on the laser as well as on the target parameters $\underline{8}$. Other acceleration regimes than TNSA have been identified and investigated with simulations, as the high power lasers for these regimes are not available yet. One of the most interesting is the so called Radiation Pressure Acceleration regime (RPA), which is different for thick (hole boring) and thin (light sail) targets 9. Radiation pressure effects start to be progressively more important at very high intensities, and finally they dominate over TNSA at laser intensities $I>10^{23} \mathrm{~W} / \mathrm{cm}^{2}[9$. The RPA regime could be achieved within the ELI-Beamlines project, the main goal of which is to develop 1-10 PW class lasers with very short pulses (few tens of femtoseconds) with excellent shot-to-shot reproducibility in order to produce short X-ray sources and accelerate particles in the ultra-relativistic energy range [10].

\section{ELI-BEAMLINES AND ELIMED}

ELI (Extreme Light Infrastructure) is a new type of European large scale infrastructure developed within the European ESFRI process. It is based on four laser facilities located in four different countries. One of the facilities is being developed in Prague (Czech Republic) and it is named ELI-Beamlines. ELI-Beamlines, as defined in the ELI White-Book [10], will also be 
dedicated to applications of laser produced photon and particle beams in various fields, from high-resolution $\mathrm{X}$-ray imaging to hadrontherapy. One of the goals of ELI-Beamlines is to develop and realize a transport beam line to demonstrate the clinical applicability of laser driven protons. Current hadrontherapy centers are based on conventional accelerators, huge and expensive machines, in terms of both cost and human resources. Laser-based accelerators could be a competitive alternative, as they can be smaller in size and less expensive. The design of miniaturized systems has been proposed in the literature [11, in which the laser-driven ion part is combined with a conventional beam transport system. In this framework, and with the aim to develop a new approach in hadrontherapy based on laser-driven ions, the ELIMED (Medical application at ELI-Beamlines) taskforce has been launched by a group of ELI-Beamlines and INFN researchers. The project officially started with a Memorandum of Understanding (MoU) signed between ELI-Beamlines and INFN-LNS. The purpose of the $\mathrm{MoU}$ is to start a research program aimed mainly at studying, designing and realizing an irradiation facility for dosimetric and radiobiological studies with the high energy proton/ion beams which will be produced at ELI-Beamlines [12. LNS researchers have gained experience at CATANA (Centro di AdroTerapia e Applicazioni Nucleari Avanzate), the first Italian protontherapy facility [13, 14. At CATANA a $62 \mathrm{MeV}$ proton beam, accelerated by a superconducting cyclotron, is used for treating of shallow tumors such as those in the ocular region. In the CATANA facility the clinical activity is still ongoing, and more than 200 patients have been treated since March 2002. The LNS tasks involve developing of dosimetric techniques to be used for the laser-driven beams, designing and realising the transport medical beam line, and in-vitro radiobiological studies; these assignments are strictly linked with the CATANA activity. Moreover, LNS researchers are involved in beam diagnostics (using a new Thomson-like spectrometer, the first prototype of which was recently tested and will be discussed within this paper), and in energy selection in order to produce a beam with energetic and spatial characteristics as required in clinical applications.

\subsection{The ELIMED PHASES}

ELIMED is planned to be realised in four phases:

The preparatory phase (2013-2015). During this phase the ELIMED facility will be built; even if no laser beams will be available, this phase is of fundamental importance for the final design of the transport beam-line, from the target to the irradiating point. The design concerns the selection system, namely a magnetic selector able to operate with a $30-60 \mathrm{MeV}$ proton beam in order to produce a beam with a final energy spread of about $5-20 \%$. An innovative Thomson spectrometer able to deflect particles with energy up to $100 \mathrm{MeV}$ will be realised, starting from the spectrometer already developed at LNS. The in-air transport line will also be developed. A preliminary dosimetric system able to detect the beam fluence will be located along the beam line. The detectors are also planned to be tested under conventional beams at LNS and under laser-driven beams in other laser facilities.

First phase (2015-2016). The first laser beams will be available inside the ELIMED hall in order to test the selection and diagnostic systems. According to already performed Particle in Cell (PIC) simulations, proton beams up to $20-30 \mathrm{MeV}$ will be produced,

Second phase. Proton beams with energy up to $60 \mathrm{MeV}$ will be produced.

Third phase. Proton beams with energy up to $100 \mathrm{MeV}$ will be produced. The whole selection and diagnostic system will be working at its maximum performance. The transport line and the dosimetric systems will be commissioned and the final radiobiological study of the beam will be realised.

Inside the ELIMED facility an experimental hall for radiation therapy has already been assigned.

\subsection{The LNS Thomson PARABOla SPECTROMETER}

The Thomson Parabola (TP) spectrometer is a widely used beam diagnostic device. The working principle, widely described in the literature [15-18], is based on parallel electric and magnetic fields acting on a well collimated ion beam propagating orthogonally to the fields themselves. The Lorentz force splits the different ion species of the beam according their $q / m$ ratio. This deflection on the detection plane results in a set of parabolic traces, each of them corresponding to a well determined ion species. Moreover the electric and magnetic deflection, mutually perpendicular, are related to the energy and momentum of the particles. Hence several types of information can be simultaneously extracted from a spectrogram. A new model of TP has been developed at LNS. It will permit the detection of high energy protons (up to $20 \mathrm{MeV}$ for protons) and wide acceptance (of the order of $10^{-4} \mathrm{msr}$ ). Its deflection sector is made of partially overlapping electric and magnetic fields in order to have a more compact system. The electric field is produced by two copper electrodes, and the magnetic field is due to to an electromagnet with $\mathrm{H}$-shaped iron. The resistive coils allow the dynamics of the spectrometer to be increased while the H-shaped iron ensures high field uniformity despite the big gap between the coils. The new technical solution is better than the previous solution based on permanent magnets. Indeed, when permanent magnets are used, the dynamics is constrained and the field uniformity requires a small gap, which affects the spectrometer acceptance. Figure 1 shows the field profile along the direction of particle propagation. 


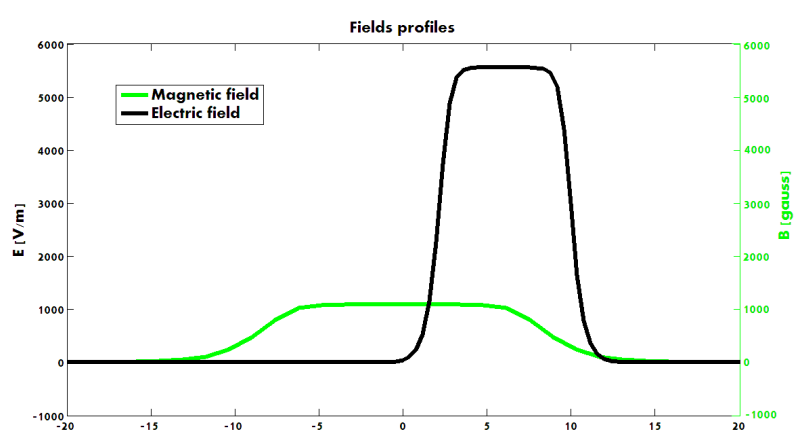

FiguRE 1. Electric and magnetic field profile along the direction of particle propagation.

The maximum achievable magnetic field intensity is 2500 gauss, corresponding to a coils current of $86.2 \mathrm{~A}$. For current values higher than $65 \mathrm{~A}$, a cooling system is necessary in order to avoid outgassing problems. The geometrical magnetic length is $15 \mathrm{~cm}$ but its effective length is $18.82 \mathrm{~cm}$. The copper electrodes that produce the electric field are $7 \mathrm{~cm}$ long (effective length $8.04 \mathrm{~cm}$ ) and they start at $6 \mathrm{~cm}$ from the magnetic field centre. A scheme of the deflection sector is available in 13 , 14. The collimator, a $10 \mathrm{~cm}$ long cylinder with two pinholes of $1 \mathrm{~mm}$ and $100 \mu \mathrm{m}$, respectively, is located upstream the deflection sector. Downstream the deflection sector, after about $40 \mathrm{~cm}$ of drift, the imaging system for particle visualization is mounted. It is constituted by a microchannel plate detector in double Chevron configuration, coupled to a phosphor screen and to a conventional reflex camera that is remotely controlled.

\subsection{Design study of An Energy Selector}

In view of the potential use of laser-driven protons for medical applications, a magnetic system is under study in order to energetically select the particle that are produced. The device consists of four dipoles based on permanent magnets producing a field of 0.7 T each (see Figure 3). The second and the third magnetic fields are parallel whit each other, but are oriented antiparallel to the first and the fourth field. This configuration allows an increase in the separation between the particle trajectories at different energy corresponding to the central pair of magnets where, by means of a slit device, particles with suitable energy are selected. The energy spread and the number of particles passing through the slit depends on the size of the aperture. The lower the energy spread, the lower the number of particles that will be transported through the energy selector, because it needs to use a smaller slit size, and vice versa (see Figure 2). The energy of the proton beam can be tuned by moving the slit position transversely between $30 \mathrm{~mm}$ and $8 \mathrm{~mm}$ from the target normal axis. The roller guide system where the central twin magnets are placed, allows the two magnets to be displaced radially in order to increase the transversal displacement and select the lowest energy particles. In this way, the energy could

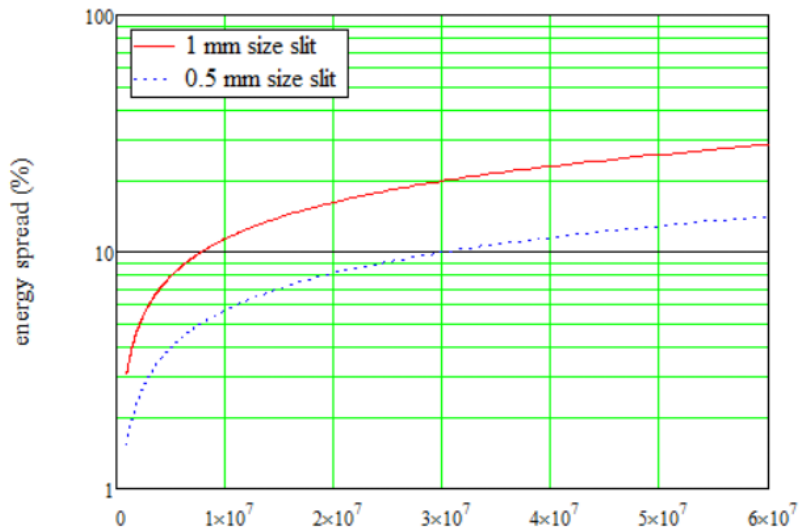

FiguRE 2. Calculated energy spread as a function of the energy of the selected beam. The curves correspond to the values obtained by using two sizes of the slit $(0.5$ and $1 \mathrm{~mm})$.

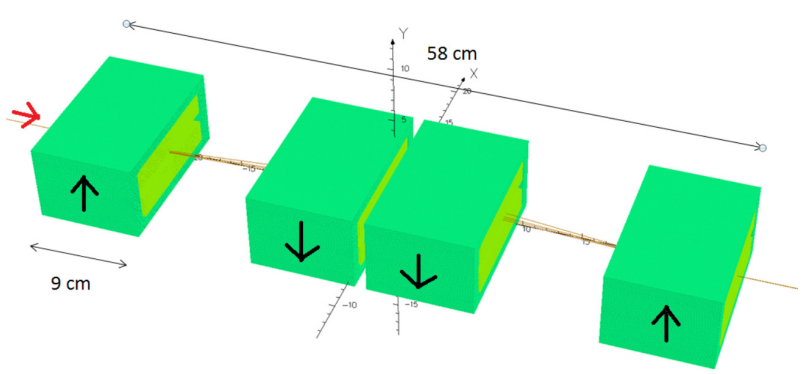

FiguRE 3. Layout of the magnet system used as an energy selector for the beam produced by the lasertarget interaction.

vary within the wide range of $5 \mathrm{MeV}$ and $50 \mathrm{MeV}$. The energy spread reachable using a $1 \mathrm{~mm}$ slit aperture ranges from $3 \%$ for low energy $(1 \mathrm{MeV})$ up to $30 \%$ for the highest $(60 \mathrm{MeV})$. The whole magnet system is almost $600 \mathrm{~mm}$ long and will be placed into a dedicated vacuum chamber. Two additional collimators are placed both upstream and downstream the selector system in order to control the proton beam size.

\section{EXPERIMENTAL RESULTS}

The TP has been tested under laser-driven beams at the PALS (Prague Asterix Laser System) laboratory using the Asterix IV laser. It has a long pulse, $(\sim 300 \mathrm{psec})$ and supplies energy up to $1 \mathrm{~kJ}$ at the fundamental wavelength of $1.315 \mu \mathrm{m}$ [19, 20]. During the measurement session, the laser energy range was between $453 \mathrm{~J}$ and $516 \mathrm{~J}$. The TP has been connected to the interaction chamber at 180 degrees with respect to the laser direction, just behind the target. An Ion Collector Ring (ICR) has been located upstream the $\mathrm{TP}$ and the distance between the target holder and the ICR was $108 \mathrm{~cm}$. Another Thomson Parabola, developed at PALS, was located at 30 degrees with respect to the laser incidence direction. This second $\mathrm{TP}$ has a different layout, as the fields are in series and the distance between the magnetic field end and 


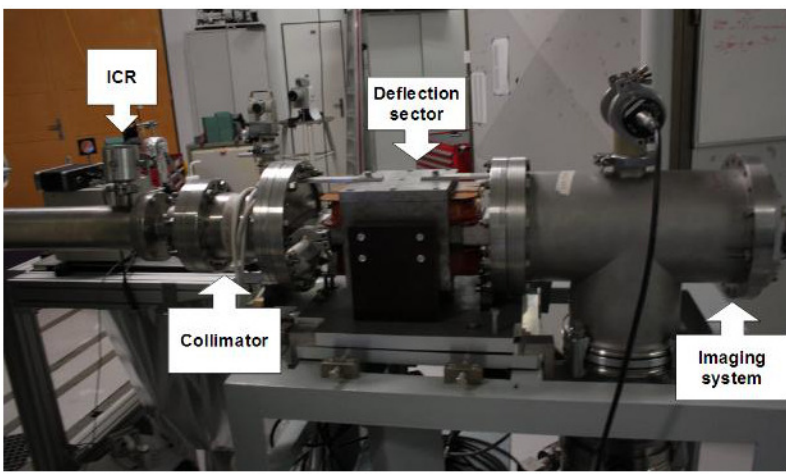

Figure 4. View of the Thomson Parabola and ICR at the PALS laboratory.

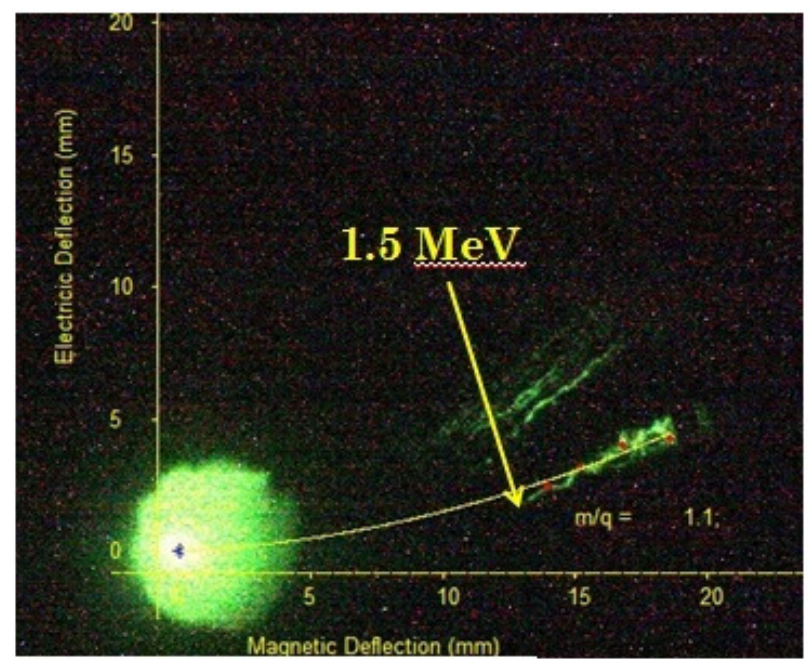

FiguRE 5. Spectrogram analyzed using the MATLAB analysis tool.

the electric field is $1 \mathrm{~cm}[21,22$. An ICR has been set upstream the collimating system of this TP (distance target-ICR $151.7 \mathrm{~cm}$ ).

Figure 4 shows the LNS TP mounted in the interaction hall of the PALS facility during the experimental tests.

The data acquired during the measurement campaign at PALS have been analysed with a MATLAB based semi-automatic analysis tool, developed at LNS. This tool is able to fit parabolas in spectrograms calculating the q-over-m ratio and the maximum particle energy corresponding to the starting point of the trace. An analysed spectrogram, corresponding to laser shot number 42702, is shown in Figure 5 The electric and magnetic fields of the TP were set to $333 \mathrm{kV} / \mathrm{m}$ and $0.0234 \mathrm{~T}$, respectively. Maximum proton energy of 1.5 MeV has been detected in the forward direction, with the laser irradiating a 0.65 micron gold target. Gold ion parabolas are also present on the spectrogram, even if they have been not discussed in this paper.

A first check of the above results can be performed using the MATLAB-based simulation tool that we have developed. It can track the particle motion in

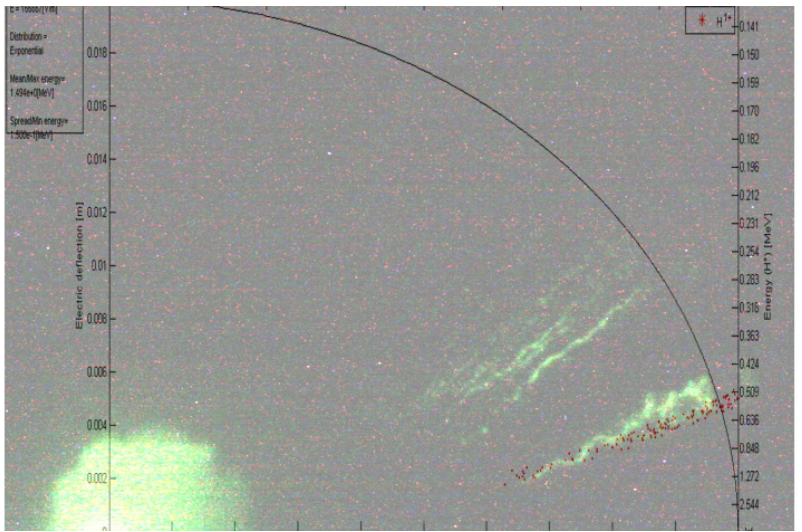

Figure 6. Spectrogram acquired using thin gold target and comparison with simulation for proton.

\begin{tabular}{ccc}
\hline & TPs & ICRs \\
\hline LN $\left(180^{\circ}\right)$ & $1.5 \pm 0.25 \mathrm{MeV}$ & $1.53 \mathrm{MeV}$ \\
\hline PalS $\left(30^{\circ}\right)$ & $1.1 \pm 0.011 \mathrm{MeV}$ & $1.13 \mathrm{MeV}$ \\
\hline
\end{tabular}

TABLE 1. Experimental results - maximal $H^{+}$energy.

the TP solving the differential equations of motion. Computational errors are reduced using the ode23 solver, included in the MATLAB ode suite, and based on the Stromer-Verlet integration scheme. This ensures energy conservation for particles moving in a magnetic field [23]. A comparison between experimental and simulated proton parabolas for the shot under consideration is shown in Figure 6 .

The data acquired with the TP has been further checked using an ICR coupled with a fast digital oscilloscope, which provides information on the beam components Time-Of-Flight (TOF). The proton peak is usually identified just after the narrow X-Ray photopeak. Thus, once the proton peak is located, it is possible to obtain information on the corresponding TOF [24, 25]. The TOFs from the ICR signals and the maximum energy estimation from the spectrograms are in good agreement. The PALS TP data has been analysed with the same approach. In this case, lower proton energies are detected, in agreement with the backward direction [26] of the system position. For the shot under consideration, maximum energy values and the average TOF values are shown in Tab. 1. Data from IRCs are considered without errors. Consistency between the ICR signal and the TP result is evident.

\section{Conclusions}

The first experimental run performed with the LNS $\mathrm{TP}$ has shown that the system is functioning well, also in comparison with other ion detectors, although further improvements are required. A new, off-axis and more precise collimation system is under study: it will allow better beam collimation, and a more reliable alignment procedure, an increase of the imaging detector field of view. 
The improved spectrometer will be tested with the cyclotron beam at LNS to perform energy calibration and to verify the maximum energy limit. The spectrometer will also be upgraded in order to detect protons with energy up to $100 \mathrm{MeV}$ available within the future ELIMED beamline.

\section{ACKNOWLEDGEMENTS}

This work has been performed in the framework of the LILIA (Laser Induced Light Ion Acceleration) project funded by INFN and in the framework of the Ministry of Education, Youth and Sports the Czech Republic's support for ELI-Beamlines (CZ.1.05/1.1.00/483/02.0061), OPVK (CZ.1.07/2.3.00/20.0087) and OPVK (CZ.1.07/2.3.00/20.0279), and within the activities of the Fyzikalni ustav, AV CR, v.v.i. We offer our special thanks to the staff of PALS for their support during the experimental measurements.

\section{REFERENCES}

[1] Wilks, S. C. et al.: Energetic proton generation in ultra-intense laser-solid interactions. Phys. Plasmas $\mathbf{8}$, 542 (2001); DOI:10.1063/1.1333697

[2] Pucella, G., Segre, S. E.: Fisica dei Plasmi. Zanichelli editore S.p.A, Bologna, 2010.

[3] Eliezer, S.: The Interaction of High-Power Laser with Plasmas. IOP Publishing Ltd, 2002.

[4] Gibbon, P., Short Pulse Laser Interaction with Matter. Imperial College Press, 2005.

[5] Passoni, M. et al.: Target normal sheath acceleration: theory, comparison with experiments and future perspective. New J. Phys. 12045012 (2010); DOI:10.1088/1367-2630/12/4/045012

[6] Joshi, C., Katsuleas, T.: Plasma Accelerators at the Energy Frontier and on Tabletops. Phys. Today 56(6), 47 (2003); DOI:10.1063/1.1595054

[7] C. T. Zhou, C. T., He, X. T.: Intense laser-driven energetic proton beams from solid density targets. OPTICS LETTERS, Vol. 32, No 16, August 15, 2007; DOI:10.1364/OL.32.002444.

[8] Malka, V. et al.: Practicability of protontherapy using compact laser systems Med. Phys. 31, 1587(2004); DOI:10.1118/1.1747751.

[9] Macchi, A., Benedetti, C.: Ion acceleration by radiation pressure in thin and thick targets. Nucl. Inst. Meth. Phys. Res. A (2010) DOI:M10.1016/j.nima.2010.01.057

[10] ELI - Extreme Light Infrastructure. Science and Technology with Ultra-Intense Lasers: WhiteBook.

[11] Sakaki, H. et al.: Designing integrated laser-driven ion accelerator system for hadron therapy at PMRC (Photo Medical Research Center). Proceedings of PAC09, Vancouver, BC, Canada TU6PFP009.
[12] INFN, FZU: ELIMED: Memorandum of Understanding.

[13] Cirrone, G. A. P. et al: Diagnostic for the radiotherapy use of laser accelerated proton beams. Radiation Effects and Defects in Solids, Vol 00, No. 00, January 2008, 1-7; DOI:10.1080/10420151003731942

[14] Maggiore, M. et al.:Design and realisation of a Thomsom Spectrometer for Laser Plasma Facilities.Nejaka Hlavicka 123(2020), 123-456.

[15] Rhee, M. J.: Compact Thomson spectrometer., Rev. Sci. instrum. 55 (8), August 1984; DOI:10.1063/1.1137927

[16] Schneider, R. F., Luo, C. M., Rhee, M. J.: Resolution of the Thomson spectrometer. J. Appl. Phys. 57 (1), 1 January 1985; DOI:10.1063/1.335389

[17] Harres, K. et al.: Development and calibration of a Thomson parabola with microchannel plate for the detection of a laser-accelerated MeV ions. Rev. Sci. Instrum. 79, 093306 (2008); DOI:10.1063/1.2987687

[18] Jung, D. et al.: Development of a high resolution and high dispersion Thomson parabola. Review of Scientific Instruments, 82(1):013306, 2011; DOI:10.1063/1.3523428

[19] Jungwirth, K. et al.: The Prague ASTERIX Laser System. Physics of Plasmas, Volume 8, number 5, May 2001; DOI:10.1063/1.1350569

[20] Margarone, D. et al.: New methods for high current fast ion beam production by laser-driven acceleration. Review of Scientific Instruments 83, 02B307 (2012); DOI:10.1063/1.3669796

[21] Torrisi, L. et al.: Proton emission from laser-generated plasmas at different intensities. NUKLEONIKA 2012;57(2):237-240.

[22] Torrisi, L. et al.: Proton emission from thin hydrogenated targets irradiated by laser pulses at $1016 \mathrm{~W} / \mathrm{cm}^{2}$. Rev. Sci. Instrum. 83, 02B315 (2012); DOI:10.1063/1.3673506

[23] Shampine, L. F., Reichelt,M. W.: The MATLAB ODE Suite. SIAM Journal on Scientific Computing, Vol. 18, 1997, pp 1-22.

[24] Margarone, D. et al.: Full characterization of laseraccelerated ion beams using Faraday cup, silicon carbide and single-crystal diamond detectors. Journal of Applied Physics 109, 103302 (2011); DOI:10.1063/1.3585871

[25] Margarone, D. et al.: Real-time diagnostic of fast light ion beams accelerated by a sub-nanosecond laser.Nukleonika 2011; 56(2):136-141.

[26] Psikal, J. et al.: Ion acceleration by femtosecond laser pulses in small multispecies targets. Physics of Plasmas 15, 053102 (2008); DOI:10.1063/1.2913264 